\title{
Quaderni
}

QUADERN I Communication, technologies, pouvoir

69 | Printemps 2009

Universités sous influence du numérique et du management

\section{Universités et ressources numériques : une affaire entre acteurs publics}

Pierre Mœglin et Françoise Thibault

\section{(2) OpenEdition}

Journals

Édition électronique

URL : http://journals.openedition.org/quaderni/311

DOI : 10.4000/quaderni.311

ISSN : 2105-2956

Éditeur

Les éditions de la Maison des sciences de l'Homme

Édition imprimée

Date de publication : 1 mai 2009

Pagination : 27-40

Référence électronique

Pierre Mœglin et Françoise Thibault, « Universités et ressources numériques : une affaire entre

acteurs publics », Quaderni [En ligne], 69 | Printemps 2009, mis en ligne le 05 avril 2012, consulté le 20 avril 2019. URL : http://journals.openedition.org/quaderni/311 ; DOI : 10.4000/quaderni.311

Tous droits réservés 


\section{$D$ ossier}

\section{universités \\ et ressources \\ numériques : \\ une affaire entre \\ acteurs publics \\ Pierre
Mœglin}

LabSic, Université Paris 13

Maison des Sciences de

l'Homme Paris Nord

Françoise Thibault

Fondation Maison des Sciences de l'Homme de Paris
Il ne suffit pas de constater que la question des politiques publiques fait souvent l'objet d'un traitement insuffisant dans les recherches sur l'introduction dans l'enseignement supérieur des systèmes d'information et de communication et des ressources numériques en général (Thibault, 2008) pour saisir ipso facto la signification et les enjeux de ces politiques publiques.

Il faut d'abord se poser la question de leur existence et de leurs conditions de possibilité. C'est-à-dire se demander, ainsi que nous le ferons en une première étape, dans quelle mesure les dispositions prises en France depuis les années 1980 par les pouvoirs publics, État, collectivités et responsables au sein des universités, en vue de développer la production et les usages de ces systèmes et ressources multimédias dans l'enseignement supérieur, correspondent à ce que l'on entend par politiques publiques. Ensuite, nous nous demanderons à quel ensemble de décisions institutionnelles dotées de quelle cohérence ces politiques obéissent, déterminées par quels principes directeurs (ou référentiels), partagés par quel ensemble d'acteurs, et se traduisant par quelles mesures concrètes pour une période donnée. Tel sera l'objet de notre seconde étape. Nous conclurons sur le constat que les politiques de mise en ligne des ressources universitaires sont coproduites par des acteurs publics en dehors de toute intervention des acteurs privés, éditeurs et industriels de la communication et des médias ${ }^{1}$.

\section{Penser l'agenda des décisions ministérielles}

Quiconque étudie les décisions ministérielles concernant la numérisation de l'enseignement supérieur est, au départ, saisi et un peu désarçonné 
par la multitude des domaines et par la disparité de leurs enjeux. De fait, la numérisation concerne aussi bien la documentation et les bibliothèques que la gestion informatisée des moyens financiers, humains et matériels des universités, le volet «Technologies de l'information et de la communication pour l'éducation » (Tice) dans les contrats d'établissement que la mise en ligne des revues, les prêts bancaires consentis aux étudiants pour l'achat d'ordinateurs personnels et bien sûr le soutien à l'enseignement à distance.

Cependant, même si ces domaines sont très hétérogènes, l'observateur finit quand même par percevoir qu'un seul et même projet s'y retrouve à chaque fois : il s'agit grosso modo de profiter de la mise en place des infrastructures numériques pour promouvoir des modes de fonctionnement décloisonnés, plus flexibles, plus réactifs, seraitce au détriment des positions scientifiques et pédagogiques acquises, des pouvoirs existants et des compétences reconnues par l'institution. Ensuite, évidemment, ces remises en cause jouent dans des sens différents, selon les cas et les rapports de force, privilégiant des points de vue réactionnaires ou innovateurs, à supposer que ces classifications gardent leur signification dans les contextes actuels.

Tel est aussi, avec toutes ses incertitudes afférentes, le projet qui détermine les décisions concernant la numérisation des ressources pédagogiques. Une fois ce premier constat établi, le foisonnement des initiatives et décisions saute à nouveau aux yeux, obligeant le chercheur à procéder à une sélection des faits lui apparaissant les plus significatifs. Ce sera l'objet de cette première étape : procéder à cette sélection et examiner dans quelle mesure les faits retenus rentrent dans la catégorie des politiques publiques.

\section{Balises}

Trois dates, correspondant à trois initiatives ministérielles, sollicitent plus particulièrement notre attention, pour la fonction de balises qu'elles offrent à l'analyse. La première est la création du Réseau Universitaire des Centres d'Autoformation (Ruca) en 1987 ; la deuxième est le lancement des campus numériques en 2001 ; la troisième est l'annonce et la mise en chantier des Universités Numériques Thématiques (UNT) en 2003.

En quoi ces trois dates font-elles «balises »? Aucune d'entre elles ne saurait être coupée des deux autres ni du contexte dans lequel elle se situe. Aussi, comme cela apparaîtra plus bas, chacune est-elle porteuse d'un ensemble de déterminations qui lui sont propres, mais sans que cela ne l'empêche de contribuer avec les autres à la construction de ce qui, rétrospectivement, se présente comme des politiques publiques. Autrement dit, ces dates sont des balises en ce que, mieux que d'autres ${ }^{2}$, elles jouent un double rôle de jalons sur une trajectoire et de sémaphore par rapport à des arrière-plans qu'elles révèlent et cristallisent.

Le lancement du Ruca mérite, à cet égard, une mention particulière : non seulement, à l'instar des deux opérations qui le suivent, il offre un exemple significatif de l'association qui vient d'être évoquée entre inscription dans une continuité et singularité idiosyncrasique, mais encore, en tant qu'opération inaugurale, il met en place des éléments qui, combinés autrement, 
se retrouveront dans les opérations ultérieures et qui se définissent par rapport à lui, en positif comme en négatif.

Le Ruca s'inscrit en effet dans la logique du Plan « Informatique pour Tous » (IPT) du supérieur lancé en 1985 par le ministère, dont il applique certains principes à l'enseignement de quatre disciplines - informatique, mathématiques, physique et sciences de la vie pour le premier cycle de l'époque ( $\mathrm{Bac}+1$ et $\mathrm{Bac}+2)$-, à l'initiative de cinq universités, Bordeaux 1, Grenoble 1, Lille 1, Nancy 1 et Paris 6, auxquelles d'autres s'ajouteront ensuite, en vue de la production et de l'intégration dans la pédagogie de ressources conçues par des enseignants « de base ». Ces enseignants, il faut le signaler, n'ont été sélectionnés par aucun appel d'offre et ils ne bénéficient d'aucun statut particulier d'auteurs. Leur production est destinée à leur usage personnel, à celui d'autres enseignants (pendant les cours) et à celui des étudiants, dans les salles d'auto-formation ou à leur domicile.

Descendant d'IPT, le Ruca hérite aussi de tout ce qui a motivé IPT. Ce plan est en effet une retombée du rapport de Simon Nora et Alain Minc (1978) sur « l'informatisation de la société » et de celui de Jean-Claude Simon (1981) sur « l'informatisation de l'école », lequel consacre des développements à l'enseignement supérieur, bien qu'il s'intéresse principalement à l'enseignement secondaire. Ainsi précédé par ces importantes contributions à l'élaboration d'une action gouvernementale en matière de « télématique » et au débat public qui l'accompagne, «Informatique pour tous » est la première initiative publique visant à développer la production et les usages des ressources informatiques dans l'enseignement supérieur.

Ce n'est pourtant pas la première fois que l'État favorise le développement d'outils et de médias éducatifs : avant IPT et le Ruca, d'autres opérations touchent prioritairement l'enseignement secondaire et concernent l'audiovisuel, les réseaux et les « nouvelles technologies ». Ce sont, entre autres, « Jeunes Téléspectateurs actifs » en 1979-1980, «Audiovisuel pour tous » en 1987, «Educâble » entre 1987 et 1998, ainsi que les programmes de câblage des établissements et le Plan « Nanoréseaux » au début des années 1990. Antérieures au Ruca, contemporaines et postérieures, ces opérations font toutes partie, selon des modalités qui leur sont propres, des initiatives ministérielles visant à assurer et à confirmer l'État dans son rôle d'initiateur des innovations technico-pédagogiques.

Cependant, elles mettent aussi l'accent sur deux éléments supplémentaires dont, plus que d'autres, le Ruca porte la marque : d'une part, la priorité donnée aux ressources à produire et à utiliser ou, plus exactement, à produire en les utilisant et à utiliser en les produisant; d'autre part, l'importance accordée à la localisation d'opérations qui, pour être nationales, n'en sont pas moins ancrées territorialement.

Tel est, brièvement esquissé, le contexte dont, dès sa création, le Ruca est tributaire : intervention étatique, priorité à la production-utilisation des ressources et territorialisation des initiatives.

Si ces trois ingrédients se retrouvent dans l'opération « campus numériques », puis dans le lan- 
cement des universités numériques thématiques, la recette n'y est plus tout à fait la même. En effet, l'appel à projets «campus numériques » privilégie, dans un premier temps au moins, l'intégration des ressources et non leur production. Plus exactement, il exprime implicitement des réserves par rapport aux démarches (typiques de celle du Ruca) centrées sur la capitalisation de ressources en ligne, alors que le volume de leurs utilisations reste relativement bas. Néanmoins, puisque des utilisations plus intensives de ces ressources supposent une activité éditoriale, ne serait-ce que pour post-produire les ressources qui existent déjà en les réagençant en fonction des besoins de publics spécifiques, la question de la production n'est quand même pas tout à fait laissée de côté. Ainsi voit-on comment, du point de vue des campus numériques, le Ruca se pose à la fois en modèle et en anti-modèle.

Le même mélange de filiation et de rupture intervient à nouveau entre campus numériques et universités numériques thématiques, comme cela apparaîtra plus bas. De la complexité de ces situations il faut donc prendre la mesure aussi exactement que possible si l'on veut saisir les raisons et déterminants de politiques qui, à l'analyse, ne se révèlent ni si erratiques que les apparences pourraient le laisser croire, ni si cohérentes que ne le ferait penser la succession des initiatives.

\section{Constructivisme}

Comment mener cette analyse ? En commençant par rompre avec la technique des check lists implicitement destinées à des décideurs et, pour ce faire, adopter les principes pratiqués par des chercheurs comme Parsons, Thoenig, Muller ou Jobert, dont l'analyse des politiques publiques se situe aux antipodes des travaux américains des années 1950, centrés sur la détermination des critères fondant une « bonne » ou une « mauvaise » politique. Écartant l'idée qu'une politique soit un processus de décision abstrait dont le sens pourrait être saisi de l'extérieur à partir d'un ensemble de contraintes économiques et historiques, nous substituons aux notions de « pouvoir public » et de « gouvernance », celle de «policy making». C'est là, en effet, une manière de marquer que la politique s'incarne à des degrés divers dans des acteurs ou des groupes d'acteurs dont les stratégies et leurs ressorts sont à appréhender. Et ce, sans pour autant attribuer à ces acteurs une liberté qu'ils n'ont pas vis-à-vis du cadre d'action préexistant, en l'occurrence celui de l'institution universitaire.

$\mathrm{Au}$ nom du principe selon lequel les politiques publiques ne sont pas des faits donnés a priori mais des phénomènes socialement construits, nous reprendrons les cinq critères identifiés par Yves Mény et Jean-Claude Thoenig (1989) pour attribuer aux initiatives qui nous intéressent le statut de politiques publiques : celles-ci doivent être constituées de mesures concrètes, elles se traduisent par des décisions de nature plus ou moins autoritaire, elles s'inscrivent dans des cadres généraux d'action, elles ont des destinataires et enfin elles définissent des buts ou des objectifs à atteindre. Dans le prolongement des recherches menées sur le Ruca (Thibault, 2003, Combès et Mœglin, 2005) et sur les campus numériques (Thibault, 2007), nous partirons de ces cinq critères en les appliquant aux trois cas qui nous intéressent de façon à nous interroger plus 
particulièrement sur les contours de ces politiques et sur les réseaux d'acteurs qui participent à leur élaboration, sur le fonctionnement du milieu décisionnel lui-même et sur les processus de changements de référentiels intervenus en cours de route (Jobert, 1994).

\section{Sens et objectifs des politiques de numéri- sation}

Puisque, telle qu'elle vient d'être signalée, la récurrence des mêmes ingrédients, de programme en programme, donne du poids à l'hypothèse selon laquelle, à travers les trois balises qui viennent d'être signalées, des politiques publiques sont bel et bien mises en œuvre en faveur de la numérisation de l'enseignement supérieur, la question est de savoir maintenant dans quel sens ces politiques jouent et quelle est leur portée. Autrement dit : au service de ces politiques, quels objectifs avec quels moyens ?

\section{Ruca : trois politiques pour un réseau}

Ainsi qu'il a été indiqué précédemment, les politiques publiques ne sont pas le fait d'un acteur unique : aux côtés du ministère, avec lui ou contre lui, les directions des universités et les enseignants porteurs de projets participent à leur construction. Et comme, par la force des choses, les intérêts en présence ne sont pas convergents, l'orientation prise par ces politiques dépend des rapports de force entre les acteurs impliqués dans leur construction.

Pour ce qui le concerne, le Ruca se situe à michemin entre deux pôles : celui du volontarisme tutélaire - qui est d'ailleurs partagé à des degrés divers par des acteurs situés à différents endroits et niveaux de l'institution - et celui de l'autogestion coopérative. De la tension entre ces deux pôles le Ruca porte la marque davantage que les opérations ultérieures. Surtout, entre ces deux pôles, nous allons le voir, trois politiques différentes s'élaborent successivement.

Lorsqu'en 1987, l'existence du Réseau universitaire des centres d'autoformation est officialisée, à l'initiative de la Division de l'éducation permanente de la Direction des enseignements supérieurs au ministère de l'Éducation nationale, de la jeunesse et des sports, l'accueil favorable que cette officialisation reçoit dans plusieurs universités et le soutien que le ministère accorde au Réseau ont une conséquence immédiate : le lancement d'un programme de deux ans reconductibles, financé par la tutelle, rompant avec la situation antérieure, dans laquelle les innovateurs dépendaient de financements toujours susceptibles d'être remis en question pour des raisons extérieures à leur activité même. En cette occasion, se mettent donc en place les conditions sine qua non d'une véritable politique, bénéficiant d'une continuité et d'une visibilité qui, sinon, lui auraient fait défaut.

L'on doit alors se demander dans quel sens le rapport des forces s'établit entre la tutelle et les acteurs de terrain ainsi qu'au sein de ces acteurs. Pour autant qu'il est possible de reconstituer après coup l'évolution de la situation, trois éléments méritent d'être pris en considération, correspondant, chacun respectivement, à l'une des trois politiques qui se sont succédées.

Le premier élément milite en faveur de la supério- 
rité des pionniers présents sur le terrain. Ceux-ci bénéficient du poids des cohésions disciplinaires, lesquelles comptent beaucoup en effet, et des solidarités qu'avant l'intervention du ministère, plusieurs années de compagnonnage ont créées. Ces cohésions et solidarités comptent d'autant plus qu'au premier rang des disciplines concernées, figure l'informatique dont la communauté scientifique et enseignante est, en matière de Tice, la première à élaborer des cadres théoriques et méthodes de travail reconnus à l'échelle internationale. C'en est assez pour permettre à ses représentants de revendiquer une autorité à la fois par rapport au ministère et par rapport à leurs pairs.

Dès le début des années soixante, les recherches sur « l'éducation cybernétique » (Couffignal 1963 ) et le behaviourisme pédagogique, en provenance des États-Unis et des pays soviétiques (Quéré 2002), mettent en effet l'accent sur la modélisation des connaissances et l'activité du sujet apprenant. Le terrain est donc favorable à un projet dont les porteurs se parent d'une double source de légitimité : celle, disciplinaire, de la maîtrise des contenus et celle, pédagogique, de la capacité à enseigner efficacement ces contenus.

Telles sont les raisons pour lesquelles le Ruca débute par une recherche-action, associant l'ensemble des contributeurs à la mise à l'épreuve de la faisabilité d'un dispositif de production et de gestion de ressources pédagogiques. Cette recherche-action se double d'une recherchedéveloppement en informatique pédagogique, menée dans le cadre de la formation universitaire continue à l'initiative d'enseignants-chercheurs de Nancy et Paris. Si, à l'époque, ce type de recherche est relativement marginal et assez rare à l'université, il trouve dans le Ruca un milieu favorable, soutenu par la direction du Réseau et les représentants du ministère.

Sans que les deux dimensions, recherche-action et recherche-développement, ne disparaissent tout à fait par la suite, un deuxième ensemble d'éléments survient à la fin des années 1980, contribuant à introduire un changement de référentiel.

Quels sont ces éléments ? D'une part, la reprise du dossier «Ruca» par des responsables du secteur de la formation initiale au ministère ; d'autre part, sur le terrain, le remplacement à la tête du Réseau d'un directeur de service de formation continue par un professeur d'université fortement intéressé par la pédagogie du Premier Cycle universitaire. Par-delà ce qu'il y a de conjoncturel dans ces deux événements, l'on observe qu'ils renforcent la tendance, déjà présente antérieurement, favorisant la co-construction d'une nouvelle politique : celle-ci se préoccupera moins de recherche-action et de recherche-développement que des conditions optimales d'accueil et de suivi des étudiants en formation initiale, spécialement de ceux qui entrent à l'université (Thibault, 2003).

Bénéficiant très opportunément des budgets destinés à la lutte contre l'échec en premier cycle, les responsables de cette inflexion politique, au ministère et au Ruca, mettent en place des centres d'autoformation accueillant tous les étudiants et notamment ceux qui sont en difficulté. Tel est le cas de l'espace Alpha, lancé par le responsable du Ruca à l'université de Bordeaux 1 et érigé 
au rang d'opération pilote, en physique, chimie et mathématiques d'abord, puis dans d'autres disciplines.

En quelques années, le changement du Ruca prend donc corps : les centres d'autoformation se multiplient et des postes d'enseignants sont créés pour en accompagner le fonctionnement. Une banque de cours est régulièrement enrichie, également accessible à tous les membres du réseau, justifiant le lancement d'une plate-forme pédagogique. Et ce, non sans créer une certaine tension entre les tenants de cette plate-forme et ceux qui privilégient la production de ressources pour la banque de cours.

C'est au moment où ce changement se concrétise, vers 1994, qu'un nouvel élément introduit une perturbation de plus dans le processus décisionnel : la priorité nouvelle accordée à ce qui devient un troisième référentiel : «l'éducation tout au long de la vie ». Défendu par Maryse Quéré à la Direction générale de l'enseignement supérieur, ce référentiel est en lien direct avec les objectifs de «l'enseignement sur mesure ». Ce serait sortir de l'espace imparti à cet article que de rendre compte des conditions dans lesquelles le référentiel en question parvient à s'imposer. Indiquons simplement que son avantage décisif tient à ce qu'il ne perd rien des initiatives et référentiels antérieurs, mais qu'il introduit un cadre général plus extensif, permettant à des acteurs nouveaux d'opérer à leur profit une synthèse des acquis précédent et, au passage, d'introduire les perspectives dont ils sont porteurs.

En l'occurrence, ce référentiel s'appuie sur la disponibilité (déjà acquise) de grands ensem- bles de ressources utilisables par les étudiants, mais ces ensembles ne sont plus réservés aux étudiants de formation continue et aux étudiants en difficulté ou en situation d'échec : ils sont destinés à tous les étudiants de l'université, du moment que cette dernière est membre du Réseau. Parallèlement, les outils de management, platesformes, environnements numériques de travail, ainsi que Learning et Knowledge Management Systems commencent à retenir l'attention pour leur aptitude à opérer un suivi individualisé des dossiers étudiants et à fournir aux apprenants des prestations sur mesure.

L'un des effets du changement qui s'opère alors - et qui compte beaucoup dans son adoption - est d'atténuer, puis de résoudre la tension entre les deux options «plate-forme pédagogique » et « banque de cours ». Plus exactement, si l'option « banque de cours », finit par l'emporter, alors que la plate-forme pédagogique commence à trouver sa place au sein du réseau, c'est parce qu'à partir de 1994 les porteurs de cette plateforme sont affaiblis localement. Dès lors, de nouvelles alliances entre tutelle et acteurs de terrain contribuent à orienter le Ruca vers une activité éditoriale.

Au demeurant, la disponibilité de budgets et leur progression rapide et conséquente ${ }^{3}$ entre 1997 et 1999 donnent à la tutelle un poids inédit. Ces facilités dissipent également les réticences de nombre d'acteurs de terrain, qu'intéressait moins au départ la production de ressources que l'accueil des étudiants dans les centres ad hoc.

Rien n'est définitivement acquis, pourtant. Constatant que la production en ligne ne s'accompagne 
pas du volume correspondant d'utilisations par les enseignants et les apprenants, la tutelle et nombre de membres du Réseau perçoivent les risques que fait peser sur la légitimité du Ruca ce que, spontanément, ils tiennent eux-mêmes pour une sous-utilisation des ressources qui viennent d'être produites ${ }^{4}$. La création en 1999, à l'initiative du ministère, d'un Observatoire des usages destiné à inciter les enseignants à œuvrer davantage en faveur de l'intégration des ressources marque donc une certaine remise en question de la politique précédente. Et, lorsqu'en 2000, le Ruca s'achève pour laisser place à d'autres initiatives, la question de la pertinence du modèle éditorial, largement débattue au sein de cet Observatoire, reste ouverte : c'est ce modèle, certes, qui a permis une volumineuse production en ligne, pour un équivalent de 2000 heures de cours, mais c'est ce même modèle qui a échoué à atteindre la banalisation de l'usage de ces ressources en ligne qui, aussi bien pour le ministère que pour les responsables du réseau, constituait un objectif à long terme, quoique jamais formulé explicitement.

En treize ans, le Ruca aura donc connu trois politiques, fruits d'une co-construction entre la tutelle et les acteurs de terrain, visant des objectifs et ciblant des publics à chaque fois différents : formation continue, étudiants de formation initiale rencontrant ou non des difficultés, ensemble des étudiants. Et c'est la troisième politique qui, in fine, fait l'objet d'une nouvelle contestation. Sans doute, par ses initiatives et grâce à des budgets en croissance, la tutelle a-t-elle, durant cette période, acquis un pouvoir grandissant au sein du Réseau, au point d'en assurer le pilotage direct. Il n'en reste pas moins que le Ruca s'achève sur ce que l'on peut analyser rétrospectivement comme une discrète mise en question.

Peut-être paie-t-il en l'occurrence la rançon de sa faiblesse congénitale : limité dans le temps et dans l'espace, il « fixe » des velléités expérimentales, en même temps qu'il les cantonne au périmètre d'une opération aux ambitions restreintes. Tel est, en somme, l'inconvénient de son avantage : si son organisation rompt avec le modèle canonique, construit autour de l'intervention de l'enseignant, elle ne rompt pas pour autant avec les modes de production et de diffusion propres à la tradition universitaire. Se tenant volontairement en marge des industries éditoriales et peu soucieux de satisfaire à des critères économiques de rendement et d'efficacité, le Ruca constitue une sorte d'abcès de fixation expérimental, tentative volontairement partielle, limitée dans l'espace mais reconduite aussi longtemps que possible en vue d'ouvrir la communauté des enseignants à des exigences de fonctionnement en réseau, pour la diffusion et la gestion de ressources pédagogiques, mais sans s'en donner pour autant tous les moyens organisationnels nécessaires.

\section{Campus numériques: deux politiques à destination de l'ensemble des établissements}

Dans un contexte très différent et à une échelle supérieure, de semblables variations affectant pilotage et orientation des politiques se retrouvent, avec les mêmes éléments de base, lors des trois appels à projets pour les campus numériques, tandis que des rapports de force du même type associent les acteurs qui y sont impliqués (Thibault, 2007).

Ayant tiré des leçons du Ruca, la tutelle accorde 
dans un premier temps la priorité à l'extension de l'opération, à son institutionnalisation et à l'intégration des ressources. Plus exactement, l'objectif initial est qu'à la faveur de leur institutionnalisation, les campus numériques mobilisent davantage d'acteurs et facilitent une intégration plus étroite des ressources numériques dans les pratiques d'enseignement et d'apprentissage à distance.

Les moyens mis en œuvre concourent à cet objectif : entre 2000 et 2002, près de 18 millions d'euros sont consacrés aux trois appels d'offres, ce qui traduit un triplement du budget annuel. Sur les trois années, 90\% des universités françaises sont impliquées au moins dans un campus numérique, de même que 50 grandes écoles, 26 IUT, la totalité des IUFM, 35 établissements étrangers et 154 entreprises, associations et groupements. Il faut donc voir dans cette mobilisation un effet de la stratégie « d'enrôlement » voulue par les responsables du programme en accord avec le jury des trois appels d'offres. Cette stratégie explique le nombre conséquent de projets sélectionnés : sur les 130 déposés par les établissements, 77 reçoivent un soutien financier et 64 sont labellisés « campus numériques ».

Reste une ombre au tableau : la relative faiblesse financière de l'engagement public au regard de l'ambition affichée. Certes, l'augmentation du budget consacré aux Tice dans l'enseignement supérieur est importante. Toutefois, comparé au budget de l'enseignement supérieur (8,5 milliards d'euros en 2002), ce montant reste bien faible. Ce décalage entre objectifs et moyens a peut-être une explication : sans sous-estimer la nécessité de disposer des financements indispensables à la mise en œuvre des campus numériques, les por- teurs des politiques publiques mettent désormais l'accent sur l'institutionnalisation des opérations. L' « expérimentation institutionnelle » à laquelle ils collaborent consiste donc à impulser et à orienter l'innovation pédagogique dans le sens d'une banalisation des usages du multimédia éducatif et à transférer au bout de trois ans les coûts de fonctionnement et le pilotage des différents campus aux établissements eux-mêmes.

En 2001, plusieurs changements parmi les acteurs clés au ministère et à la tête des établissements impliqués dans le programme ne modifient pas fondamentalement les cadres de l'action : le troisième appel d'offre est lancé comme prévu. En revanche, le contenu de l'opération connaît une transformation notable : l'intérêt des nouveaux responsables pour les environnements numériques de travail (ENT) conduit à minorer la place accordée à l'enseignement à distance et à la formation des personnels idoines. Le troisième appel d'offres consacre par conséquent plus d'un tiers du budget au développement d'ENT, tandis que le nombre de campus soutenus financièrement passe de 77 à 32 .

Cette réduction significative inaugure une nouvelle politique, caractérisée comme la précédente par la volonté de toucher un grand nombre d'établissements. Simplement, la ré-ingéniérie de la formation n'est plus à l'ordre du jour, la priorité revenant aux outils de gestion de services et de contenus. Autrement dit, l'option «plate-forme de service » prend le pas sur les options «banque de cours » et «plate-forme pédagogique ». Ce repositionnement s'accompagne de l'arrivée d'ingénieurs en informatique, jusque-là cantonnés au développement des réseaux de communication et 
à l'informatique de gestion mais qu'enhardissent les progrès conséquents réalisés dans les domaines des réseaux et de l'informatique de gestion.

Par rapport au déroulement du Ruca, les conditions de fonctionnement du programme « campus numériques » témoignent d'une extension significative : celui-là en restait à un nombre de partenaires relativement réduit; avec celui-ci, s'opère un déploiement beaucoup plus important, favorisé par l'implication des établissements euxmêmes. En outre, la réorientation intervenue au bout de deux ans permet d'interrompre en douceur le soutien de la tutelle à plusieurs campus des années précédentes qui, parfois lancés à grand frais, n'ont pas produit les résultats attendus. En revanche, cette réorientation permet davantage encore de privilégier la conception d'outils utiles au management des établissements.

En phase avec la politique d'autonomie des universités qui prévaut désormais, le soutien des ENT perdure jusqu'à aujourd'hui, mais sous la stricte responsabilité des équipes universitaires et sans que ne soient mises en concurrence les offres techniques en présence, comme cela avait pourtant été envisagé. Plus exactement, si les incitations de la tutelle pour favoriser les collaborations entre les universités et le secteur industriel sont suivies de quelques effets au début de l'opération (quatre projets d'ENT sont retenus dans un premier temps, dont certains en relation avec des industriels), les collaborations avec les industriels sont rapidement ignorées voire contestées, ce qui permet aux structures universitaires de garder la main en privilégiant l'ENT « maison $»^{5}$. Telles sont les deux politiques successives qui, en trois ans, déterminent les collaborations entre tutelle et responsables universitaires autour des campus numériques.

Comme on le voit, les enjeux déjà présents du temps du Ruca se retrouvent ici, mais les cartes sont désormais redistribuées : alors que le Ruca était dominé par l'objectif de production et d'édition de ressources, c'est l'option « plate-forme » qui l'emporte avec la politique des campus numériques, mais il s'agit prioritairement de platesformes de services ; tandis qu'au terme du Ruca, le rapport des forces était favorable à la tutelle, ce sont les universités qui ont la main lorsque les trois années correspondant aux appels à projets sont écoulées. Enfin, quand la vitesse acquise du Ruca lui conférait une certaine stabilité, les programmes suscités par les appels «campus numériques » première période retrouvent la volatilité de certaines opérations ayant précédé le Ruca. Une constante cependant : ni le Ruca ni les campus numériques n'accordent la moindre place aux initiatives et à la participation des industriels de l'éducation, éditeurs, producteurs de multimédias, opérateurs de formation à distance, etc.

\section{Universités numériques thématiques : une politique autour de grands domaines disciplinaires}

Annoncé en 2003 et rendu effectif en 2004, le programme des universités numériques thématiques s'inscrit, si l'on suit les textes officiels, dans la continuité des campus numériques. Il est exact que son pilotage est assuré par le responsable d'un campus numérique, « l’Université Médicale Virtuelle Francophone (UMVF) » et qu'une partie des budgets précédemment destinés aux campus numériques « bascule » sur la nouvelle opéra- 
tion. Il suffit toutefois d'examiner les UNT sous l'angle de leurs contenus et des instruments de la politique pour s'apercevoir que, sur beaucoup de points, les deux opérations divergent : les UNT constituent bel et bien une troisième balise.

Ces organisations «sans murs » fédèrent de grands groupes de disciplines et rassemblent potentiellement l'ensemble des établissements supérieurs concernés afin de mettre des ressources pédagogiques numérisées à la disposition de tous les membres du réseau. Structurés autour du « noyau dur » des membres principaux, pilotés par une instance collégiale décisionnaire, elles mutualisent les ressources à l'échelle nationale en favorisant et validant la production et la diffusion des ressources conçues par les universités qui en sont membres. Ainsi s'opère, au sein des politiques publiques, le retour aux communautés disciplinaires, désormais promues interlocutrices privilégiées du ministère.

Ce qu'il y a de surprenant dans la création et le développement des UNT tient à ce que ce programme se situe en porte-à-faux par rapport aux orientations générales de la politique ministérielle laquelle, depuis la fin des années 1980, pousse à l'autonomie des établissements et à la responsabilisation de leurs dirigeants (Musselin 2001). Certes, la manière dont la tutelle est organisée, qui concède à la fois marginalité et autonomie à la Sous-direction en charge des Tic au ministère, explique pour partie la domination du référentiel traditionnel d'une communauté scientifique s'auto-organisant et se chargeant de la responsabilité de la libre circulation des savoirs. Mais cette explication est-elle suffisante?

Avant de parler d'anomalie politique, ce dont, à ce stade de notre analyse nous nous gardons bien, il conviendrait d'engager de plus amples investigations sur les structures décisionnelles. Ainsi pourrait-on vérifier si les UNT opèrent effectivement un retour en arrière, en sautant par dessus les campus numériques, pour revenir à des positions plus proches de celles qui valaient lors du Ruca. Cela semble bien être le cas si l'on en juge par la place que les UNT accordent à la production des ressources. Des réserves s'imposent toutefois, ne serait-ce que parce que, par exemple, les UNT évacuent la question du contrôle éditorial que le Ruca, à la fin de ses activités, avait clairement posée.

\section{Conclusions}

$\mathrm{Au}$ terme de ce rapide examen, quatre points méritent plus particulièrement d'être mis en lumière.

Le premier, en relation avec l'objet principal de notre recherche, a trait à la qualification des petites et grandes décisions ayant contribué à la production et à l'utilisation en ligne des ressources multimédias dans l'enseignement supérieur, entre 1987 et la fin des années 2000. Nous pensons avoir établi qu'aussi sinueuses et fluctuantes que ces décisions paraissent de l'extérieur, elles s'inscrivent dans des politiques publiques. De fait, nous avons pu vérifier qu'elles déterminent des actions concrètes, pour un certain temps et en fonction d'objectifs correspondant à des référentiels à peu près clairement affichés; elles sont le résultat d'évolutions à l'intérieur de la configuration universitaire (tutelle, établissements, profession) et le produit de coalitions entre acteurs qui, à part plus ou moins égale, contribuent à leur 
construction ; enfin, elles sollicitent les éléments d'une matrice mise en place dès l'époque du Ruca et dont les opérations suivantes reprennent les éléments tout en les agençant différemment. Ainsi avons-nous vu se constituer de grandes alternatives autour desquelles s'organisent les fluctuations du secteur: inscription locale et pluridisciplinaire / inscription nationale et monodisciplinaire ; pilotage par la tutelle / pilotage par les responsables du terrain; option « banque de cours » / option « plate-forme », etc.

Le deuxième point porte sur le rôle joué par les « balises » dans le déploiement de ces politiques. Le Ruca et, à sa suite, les programmes « campus numériques » et « Universités Numériques Thématiques » sont simultanément les conséquences de ces politiques et l'occasion de leur réactivation sur des bases renouvelées. À chaque fois, en effet, des événements tels que l'affaiblissement local d'un acteur, un changement de responsable au plan national, l'arrivée d'une équipe ayant de nouveaux centres d'intérêt, obligent à rebattre les cartes et incitent tel ou tel groupe d'acteurs à chercher à prendre l'avantage sur ses homologues. Ainsi s'expliquent la manière dont sont tranchées les hésitations du Ruca entre banque de cours, plate-forme pédagogique, production éditoriale et plate-forme de services, ou bien les conditions de la rupture entre le Ruca et les campus numériques, puis le retour partiel des universités numériques thématiques aux principes du Ruca. Les balises marquent la continuité des politiques publiques, mais cette continuité se nourrit de la discontinuité des prises de position successives au sein des configurations d'acteurs.

Le troisième point concerne les jeux d'acteurs à l'origine de ces politiques. L'usage qu'en référence aux travaux de Christine Musselin (2001), nous avons fait de notions telles que « configuration d'acteurs » et « référentiel d'action» confirme l'intérêt du modèle constructiviste. Construction ne signifie pas, toutefois, collaboration pacifique : nous n'avons pas minoré l'importance des rapports de force entre acteurs dans la détermination des politiques publiques. Cette conception agonistique aide à comprendre la combinaison d'arbitraire et de nécessaire qui caractérise ces politiques publiques : chaque groupe d'acteurs essaie évidemment de promouvoir le type de finalité dont il se réclame, mais il arrive que des alliances ou des compromis le conduisent à rallier des positions qui ne sont pas ou pas entièrement les siennes.

Le quatrième point ressort a contrario des analyses précédentes, mais il est fondamental. Il porte sur l'absence des industriels, éditeurs et opérateurs de dispositifs de management : contrairement à ce qui a été dit, notamment par les tenants du courant altermondialiste, les politiques de numérisation de l'enseignement supérieur ne fraient pas une voie royale aux éditeurs et autres industriels privés de la mise en ligne et du management. Au contraire, l'analyse de la situation française montre que, numérique, autoproduit et autoconsommé, le polycopié reste le nec plus ultra de la production pédagogique universitaire. 
$R \cdot E \cdot F \cdot E ́ \cdot R \cdot E \cdot N \cdot C \cdot E \cdot S$

COMBÈS, Yolande, MEGLIN, Pierre (2005) :

C@mpuSciences, d'un modèle industriel à l'autre, in Patrice Grevet (2005) : Modèles économiques des campus numériques, rapport de l'ERTE remis au ministère de la recherche, Direction de la technologie, 64 pages. Consultable sur http://erte.mshparisnord.org/Moeglin.pdf COUFFIGNAL, Louis (1963) : La pédagogie cybernétique, Paris, PUF.

JOBERT, Bruno (1994) : « Le retour du politique » in Bruno Jobert, dir. (1994) : Le tournant néo-libéral en Europe, Paris, L'Harmattan.

MUSSELIN, Christine (2001) : La Longue marche des universités françaises, Paris, PUF.

NORA, Simon, MINC, Alain (1978) : L'informatisation de la société, Paris, La Documentation française.

QUÉRÉ, Maryse (2002) : « Regard rétrospectif sur l'histoire du logiciel éducatif et sur la réalité du système éducatif français : un mariage difficile », in Georges-Louis Baron et Éric Bruillard (2002) : Les Technologies en éducation. Perspectives de recherche et questions vives, Paris, INRP, pp. 115-123.

SIMON, Jean-Claude (1981): L'Éducation et l'informatisation de la société, rapport au président de la République, Paris, Fayard.

THIBAULT, Françoise (2003) : «Coalitions sociales et innovation pédagogique : le cas du Réseau universitaire des centres d'autoformation ", in Brigitte Albero, dir. (2003) : Autoformation et enseignement supérieur, Paris, Hermès / Lavoisier, pp. 193-218.

THIBAULT, Françoise (2007) : « Campus numériques : archéologie d'une initiative ministériel- le » in Études de communication, L'intégration du numérique dans les formations du supérieur, pp. 17-48.

THIBAULT, Françoise (2008) : « Politiques publiques et Tice : ne circulez pas, il y a à voir !» in Le déploiement des Tics dans l'enseignement supérieur: évidences et tendances, Actes de l'école d'été du laboratoire Gresec, 9-12 septembre 2008 (à paraître). 


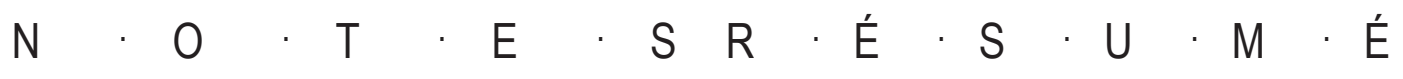

1. Précaution préalable, impossible, compte tenu des limites imparties, d'entrer dans les détails de l'analyse. Les éléments que nous soumettons à la discussion s'appuient sur des travaux réalisés par ailleurs, auxquels le lecteur est invité à se reporter pour disposer du substrat empirique.

2. Par exemple, le lancement du plan «Université numérique en région»(UNR) n'a pas été retenu comme «balise». Il ne correspond pas, en effet, à un objectif original : il s'agit seulement pour les associations entre État, régions, établissements et autres partenaires (Datar, Cnous, Crous, parfois départements et communautés d'agglomérations) de favoriser le développement des services numériques et notamment des environnements numériques de travail lancés antérieurement, lors de la seconde phase du programme " campus numériques ».

3. En deux ans, le budget alloué aux actions collectives pour les Tice, en dehors des contras d'établissements, passe de 0,3 M€ à plus de 1,5 M€.

4. Témoignage, parmi de nombreux autres, de l'un des principaux responsables du Ruca : «Les enseignants eux-mêmes vous disent : "c'est beau votre produit, quand vous nous le présentez. Mais on n'a pas le temps de s'y plonger". Donc, ils se contentent... Bon, certains l'utilisent quand même, c'est sûr, mais on a, c'est vrai, du mal à essaimer ».

5. Actuellement plus de 80 établissements sont dotés de l'ENT «Esup » développé en logiciel libre sous la responsabilité du réseau des informaticiens des universités : le CRU (comité réseau des universités).
Malgré l'impression d'hétérogénéité qu'elles donnent a priori, les décisions de ces vingt dernières années relatives à la numérisation des ressources pédagogiques universitaires et à leur mise en ligne, sont-elles suffisamment cohérentes pour que l'on soit autorisé à parler à leur propos de politiques publiques? À cette question nous répondons par l'affirmative. Nous montrons aussi que, contrairement à ce qu'on aurait pu croire, les acteurs privés, tels qu'éditeurs, opérateurs de formation à distance et concepteurs de plates-formes n'ont été associés ni à la production de ces politiques ni même à leur application. Les initiatives concernant la numérisation à l'université restent, en France, une affaire d'acteurs publics.

Even if they seem to be rather heterogeneous, are the decisions, in the last twenty years, regarding the digitalization of educational resources and their web publishing consistent enough to be considered as public policies? Our answer is yes. Moreover we argue that, despite what one could first believe, private actors, such as on-line publishers, distance learning companies and platform operators have never been involved in either the policy making in that field, or in its applications. In France, digitalization of academic resources remains a public matter. 